January, 1999

\title{
On New Conformal Field Theories with Affine Fusion Rules
}

\author{
Doron GePNER \\ Department of Physics \\ Weizmann Institute of Science \\ Rehovot 76100, Israel
}

\begin{abstract}
Some time ago, conformal data with affine fusion rules were found. Our purpose here is to realize some of these conformal data, using systems of free bosons and parafermions. The so constructed theories have an extended $W$ algebras which are close analogues of affine algebras. Exact character formulae is given, and the realizations are shown to be full fledged unitary conformal field theories.
\end{abstract}


Rational conformal field theories have been the subject of much research owing to their pivotal role in string theory and condensed matter physics, initiated in the work of Belavin, Polyakov and Zamolodchikov [1]. A relation later found by Verlinde [2], connects the fusion rules and the modular matrix. In ref. [3], it was noticed the the Verlinde formula has many, as yet undescribed, solutions and it was conjectured that these are real RCFT (rational conformal field theories). Our purpose here is to describe explicitly the realizations of these data as full fledged rational conformal field theories, for some families of subcases.

We concentrate on the affine-like conformal data, as the bosonic case was completely solved in our recent publication [4]. There we gave a formula for the central charge of the affine case. These theories are labeled by an integer $q, G_{(k+g) / q}$ where $G$ is some group, $k$ is the level and $q$ is any integer strange to $g(k+g)$. The theory $G_{(k+g) / q}$ has the usual affine fusion rules [5], and the central charge,

$$
c=\frac{q k D}{k+g} \bmod 4,
$$

where $g$ is the dual Coxeter number and $D$ is the dimension of the algebra. $q=1$ is the usual affine case. Inspecting eq. (1), we notice that for $q=p(k+g)+1$, where $p$ is some integer, the central charge obeys,

$$
c(q)-c(1)=\text { integer }
$$

Thus, it is natural to suspect that for these cases $G_{(k+g) / q}$ is the same as the usual affine case, up to some bosonic system. This is, in fact, our result. More precisely, recall the parafermionic systems [6]. The affine theories are decomposed into free bosons and parafermions, whose characters are the so called string functions, $c_{\lambda}^{\Lambda}(\tau)$, in such a way that

$$
\chi^{\Lambda}(\tau)=\sum_{\lambda} c_{\lambda}^{\Lambda}(\tau) \Theta_{\lambda}(\tau),
$$

where $\chi^{\Lambda}(\tau)$ is the affine character, and $\Theta_{\lambda}(\tau)$ are the characters of a free bosonic system, propagating of the lattice $\sqrt{k} M$, where $M$ is the root lattice. Mathemat- 
ically, this formula was discussed in [7], and ref. therein, and given a physical interpretation in ref. [6].

Our strategy, is to knock off the system of bosons in eq. (3) and to replace it by another system of bosons, so as to get the theory $G_{(k+g) / q}$. To this avail, we assume that the new bosonic system has the same fusion rules as the old one. Such bosonic theories were realized fully in ref. [4] and are also labeled by some integer $\tilde{q}$, denoted by $M_{k / \tilde{q}}$, where $M$ is the root lattice. The dimension formula for the bosonic system is

$$
\Delta_{\lambda}=\frac{\tilde{q} \lambda^{2}}{2 k} \bmod Z
$$

In the pseudo-affine theories (i.e., $q \neq 1$ ), the fields have the dimensions

$$
\Delta_{\Lambda}=\frac{q \Lambda(\Lambda+2 \rho)}{2(k+g)} \bmod Z
$$

where $\rho$ is half the sum of positive roots.

Combining, now eqs (4-5), we find the following relation for the dimensions,

$$
\frac{(q-1) \Lambda(\Lambda+\rho)}{2(k+g)}=\frac{(\tilde{q}-1) \lambda^{2}}{2 k} .
$$

This relation can be fullfilled for $q-1=(k+g) p$ due to the relation $\Lambda-\lambda \in M$. Taking $\tilde{q}=k \tilde{p}+1$, we find

$$
\frac{p \Lambda(\Lambda+2 \rho)}{2}=\frac{\tilde{p} \lambda^{2}}{2} \bmod Z .
$$

This equation can easilly be satisfied, i.e., for all $p$ we can find such $\tilde{p}$.

Examples: 1) Take $G \approx S U(2)$. Then, we take $\tilde{p}=3 p$. Eq. (7) becomes, where $\Lambda=l \alpha / 2$ and $\lambda=m \alpha / 2$,

$$
\frac{p l(l+2)}{4}=\frac{\tilde{p} m^{2}}{4} \bmod Z,
$$

which is obeyed for $\tilde{p}=3 p \bmod 4$. 
2) Take $G \approx S U(N)$ where $N$ is odd. Then we take $p=\tilde{p}$. Eq. (7) becomes,

$$
\frac{p \Lambda(\Lambda+2 \rho)}{2}=\frac{\tilde{p} \lambda^{2}}{2} \bmod Z
$$

which is obeyed since $\rho \in M$ and $\Lambda-\lambda \in M$. The same holds for all algebras with odd number of center elements.

3) Take $G=S U(N)$ where $N$ is even. It can be checked that it works exactly like $S U(2)$ with $\tilde{p}=(N+1) p$. We omit the detail for brevity sake.

For all the groups it is straightforward to solve the relation eq. (7). Further, since we know all the realizations for such bosonic theories [4], it follows that we have the complete realizations for all these affine systems as full fledged unitary RCFT.

It remains, to verify the modular transformation $S: \tau \rightarrow-\frac{1}{\tau}$. This is also quite simple since for the bosonic systems we have the relation,

$$
S_{\lambda, \mu}=\exp (-2 \pi i[\Delta(\lambda+\mu)-\Delta(\lambda)-\Delta(\mu)])
$$

we find that both sides of eq. (3) have the same modular transformations. On the l.h.s. we have

$$
S_{\Lambda, \Lambda^{\prime}}=i^{|\Delta|}\left|\frac{M^{*}}{(k+g) M}\right|^{-\frac{1}{2}} \sum_{w \in W}(-1)^{w} e^{-2 \pi i q w(\Lambda+\rho)\left(\Lambda^{\prime}+\rho\right) /(k+g)},
$$

where $W$ is the Weyl group and $|\Delta|$ is the number of positive roots. For $q=$ $p(k+g)+1$ we get the phase $e^{-2 \pi i(\Lambda+\rho)\left(\Lambda^{\prime}+\rho\right) / 2}$ times the $q=1 S$ matrix, which is exactly what is needed, by eq. (10).

Now, albeit ref. [4] presents all the realizations for the bosonic theories, the solution has to be worked out case by case. Further, we would like to find the realizations with minimal central charge. Using the methods of ref. [4] it is not too difficult to present such a construction. This we do here for $S U(2)$. 
First we introduce two types of matrices [4], which depend on three integer parameters, $x, y, z$, obeying $x>0, y>0$ and $z^{2}<4 x y$. These matrices represent the scalar products between the basic vectors of the even lattices. The matrices are, of $E$ type,

$$
E_{n}^{x, y, z}=\left(\begin{array}{ccccccccc}
2 x & -z & 0 & 0 & \ldots & 0 & 0 & 0 & 0 \\
-z & 2 y & -1 & 0 & \ldots & 0 & 0 & 0 & 0 \\
0 & -1 & 2 & -1 & \ldots & 0 & 0 & 0 & 0 \\
0 & 0 & -1 & 2 & \ldots & 0 & 0 & 0 & 0 \\
\ldots & \ldots & \ldots & \ldots & \ldots & \ldots & \ldots & \ldots & \ldots \\
0 & 0 & 0 & 0 & \ldots & 2 & -1 & 0 & -1 \\
0 & 0 & 0 & 0 & \ldots & -1 & 2 & -1 & 0 \\
0 & 0 & 0 & 0 & \ldots & 0 & -1 & 2 & 0 \\
0 & 0 & 0 & 0 & \ldots & -1 & 0 & 0 & 2
\end{array}\right)
$$

for

$$
6 \leq n \leq 10, \quad \operatorname{det}\left(E_{n}^{x, y, z}\right)=\left(4 x y-z^{2}\right)(11-n)-2 x(12-n) .
$$

Of $A$ type,

$$
\begin{aligned}
A_{n}^{x, y, z}= & \left(\begin{array}{ccccccccc}
2 x & -z & 0 & 0 & \ldots & 0 & 0 & 0 & 0 \\
-z & 2 y & -1 & 0 & \ldots & 0 & 0 & 0 & 0 \\
0 & -1 & 2 & -1 & \ldots & 0 & 0 & 0 & 0 \\
0 & 0 & -1 & 2 & \ldots & 0 & 0 & 0 & 0 \\
\ldots & \ldots & \ldots & \ldots & \ldots & \ldots & \ldots & \ldots & \ldots \\
0 & 0 & 0 & 0 & \ldots & 2 & -1 & 0 & 0 \\
0 & 0 & 0 & 0 & \ldots & -1 & 2 & -1 & 0 \\
0 & 0 & 0 & 0 & \ldots & 0 & -1 & 2 & -1 \\
0 & 0 & 0 & 0 & \ldots & 0 & 0 & -1 & 2
\end{array}\right), \\
& \operatorname{det}\left(A_{n}^{x, y, z}\right)=\left(4 x y-z^{2}\right)(n-1)-2 x(n-2) .
\end{aligned}
$$


There are several cases. Introduce the usual theta function for the lattice $M$,

$$
\Theta_{\lambda}^{M}(\tau)=\sum_{\mu \in M+\lambda} e^{\pi i \tau \mu^{2}}
$$

where $M$ is some lattice and $\lambda \in M^{*} \bmod M$. First, we take for $M$ the usual parafermionic lattice $M \approx(\sqrt{2 k})$. However, we permute the fields,

$$
\chi_{l}=\sum_{m=l \bmod 2} c_{m}^{l} \Theta_{z m}
$$

we claim that for some $z$ this realizes the pseudo-affine theory, for $k=0 \bmod 8$. From the formula for the dimensions, we need

$$
z^{2}-1=\tilde{p} k
$$

which is solved as follows:

1) $k=0 \bmod 8, \tilde{p}= \pm 1 \bmod 4: \tilde{p}=k / 4 \mp 1, z=\mp 1+k / 2$.

2) $k=0 \bmod 4, \tilde{p}=2 \bmod 4: z=k \mp 1, p=k \mp 2$.

3) $k=0 \bmod 4, \tilde{p}=1 \bmod 4: M \approx E_{9}^{1, k / 4+1,1}$ or $M \approx E_{9}^{k, k / 4+1, k}$. The character is

$$
\chi^{l}=\sum_{m} c_{m}^{l} \Theta_{z m}^{M},
$$

with $z=1$. For $\tilde{p}=3 \bmod 4$ it is the same lattice with $z=k-1($ for $k=0 \bmod 8)$, $z=k / 2+1($ for $k=4 \bmod 16), z=k / 2-1($ for $k=12 \bmod 16)$.

4) For $k=2 \bmod 4, p=1,2,3 \ldots$ We have: $M \approx A_{2 p+1}^{k,(k+2) / 4, k}$ and the character is given by eq. (19) with $z=1$.

5) $k=1 \bmod 4$, with $\tilde{p}=2 \bmod 4$ we have: $M \approx E_{7}^{k,(k+3) / 4, k}$ with the character given by eq. (19) with $z=1$.

6) $k=-1 \bmod 4, \tilde{p}=2 \bmod 4: M \approx A_{3}^{1,(k+5) / 4,2}$. The characters are given by eq. (19) with $z=(k-1) / 2$. 
This concludes all the allowed cases.

Let us discuss now the extended algebra. In some cases we have, in fact, a realization in terms of affine algebra. For example, at $k=2$ all the bosonic lattices are given for all $q$ by $D_{n}$, for some $n$. The full theory is then $S O(2 n+1)_{1}$. The decomposition eq. (3) is the usual decomposition for $S O(2 n+1)$ into $n$ bosons and a fermion.

Now, in all the cases, we have an extended current

$$
X(z)=\psi_{1}(z): e^{i \alpha \phi}
$$

where $\psi_{1}(z)$ is the first parafermion of dimensions $\Delta=1-1 / k$. Thus the dimension of this current is

$$
\Delta_{X}=1-1 / k+\alpha^{2} / 2,
$$

which is always an integer. It is straight forwards to compute $\Delta_{X}$ from the list above, and we find, typically, that $\Delta_{X}=\tilde{p}+1$, where $\tilde{p}=0,1,2,3 \ldots$ In the OPE of $X$ with $X^{\dagger}$ ( $X$ with $X$ is non-singular) we find only $T$ (the stress tensor) and $J$ (U(1) current). Thus we denote by $A_{p}$ the algebra generated by $X, X^{\dagger}, T$ and $J$ (along with the derivatives and products, of course, of $T$ and $J$ ). $A_{0}$ is the usual $S U(2)$ affine algebra. $A_{1 / 2}$ (by continuation: the algebras and the models can be defined for $p$ half intgral, although the fusion rules no longer coincides with $S U(2)$ affine ones; the algebra is then $A_{p}$ with half integral $p$ ) is the $N=2$ superconformal algebra. The rest of the algebras appear to be new.

The blocks of these theories, with respect to the algebra $A_{p}$ are in 1-1 relation with ordinary $S U(2)$ obeying also the same fusion rules. The modular matrix is a permutation of the $S U(2)$ one.

The exact OPE of $X$ with $X^{\dagger}$ is easily determined. For example, for $p=1$ we find

$$
X(z) X^{\dagger}(u)=\frac{1}{(z-u)^{4}}+\frac{J(u)}{(z-u)^{3}}+\frac{a T_{p}(u)+\frac{1}{2} \partial J+\frac{1}{2} J J}{(z-u)^{2}}+
$$




$$
\frac{2 a \partial T_{p}+a J T_{p}+\frac{1}{6} J J J+\frac{1}{2} J \partial J+\frac{1}{6} \partial^{3} J}{(z-u)}+\text { regular, }
$$

where $J(u)=i \alpha \partial \phi$ is the $U(1)$ current, $a=2 \Delta\left(\psi_{1}\right) / c_{p . f .}, T_{p}=T+\frac{1}{2}(\partial \phi)^{2}$ is the stress tensor minus the $U(1)$ part. The rest of the OPE's ( $X$ and $X^{\dagger}$ with $J$ and $T$ ) are obvious and we omit them for brevity, as $X$ is a primary field with the $U(1)$ charge $\alpha^{2}$. The commutation relations for the moments of the algebra can be easily written by standard methods [1]. It is important that the algebra $A_{2}$ (as the others) is expressed solely in terms of $X, X^{\dagger}, T$ and $J$, as this guarantees its universality. The parameters above depend only on $p$ and the central charge. The representations of the algebra $A_{p}$ have two quantum numbers $\Delta$ (the dimension of the field), and $q$ (the $U(1)$ charge).

Let us digress now to describe the minimal models of the algebra $A_{p}$. These are labeled, for each $p$, by an integer $k$. The central charge is

$$
c=\frac{3 k}{k+2}
$$

i.e., the same as $S U(2)$ ( $p=0$ case). We add one free bosons to the parafermions,

$$
X=\psi_{2}^{0}: e^{i \alpha \phi}:
$$

where $\alpha=\sqrt{2(k p+1) / k}$. Now, acting $k$ times with $X$, the parafermion drops and we get a net shift in momenta of $k \alpha=\sqrt{2(k p+1) k}$. This means that the characters are expressed in terms of a theta function at the level $k(k p+1)$. Further, acting with a single $X$ gives a shift of 2 in the parafermion and a shift of $\alpha$ in the bosonic momenta. Thus, the characters are given by

$$
\chi_{m, m^{\prime}}^{l}=\sum_{r \bmod k} c_{m+2 r}^{l} \Theta_{m^{\prime}+2(k p+1) r ; k(k p+1)} .
$$

This equation holds for $p$ integer or half integer. Now, the primary fields are obtained by the demand that $X_{n}$ and $X_{n}^{\dagger}$ vanish for negative $n$. For integer $p$, we 
find

$$
\frac{\left|m-m^{\prime}\right|}{k} \leq p,
$$

and is integral. For $p$ half inetgral in the $N S$ sector it is the same, and in the $R$ sector, it is half integral. We believe that these are the minimal models for the algebras $A_{p}$ with $p=0,1 / 2,1,3 / 2,2, \ldots$.

For algebras, other than $S U(2)$, we similarly get extended algebras for the realizations. The calculation of the OPE's is more difficult, but straightforwrd.

The algebras $A_{p}$ actually can be extended to two parameters, $\alpha^{2}$ and $c$, the central charge. More general minimal models can be easily obtained by taking $X=\psi_{m}^{0} \exp (i \alpha \phi)$ for some general $m$. The discussion above, for $m=2$, readily generalizes to any $m$.

We can actually use the duality relation,

$$
S U(n)_{m} \times S U(m)_{n} \times U(1) \approx U(n m)_{1},
$$

to express the theories $S U(n)_{q}$ with $q=-1+(k+g) \tilde{q}$, in terms of $S U(m)_{n}$ parafermions and bosons. This proceeds by first writing

$$
S U(n)_{-m / q}=S U(m)_{n / q} \times \text { free bosons, }
$$

and then proceeding as before. It can be seen that the equation for the dimension of the field, eq. (6), work as before. We omit the detail.

Finally, the reader must be curious about the general pseudo-affine theory. How is it realized? We believe the answer lie in multi-parafermion theories. It was noted already by Zamolodchikov and Fateev [6] that the monodromy of parafermions

\footnotetext{
$\star$ I thank B. Noyvert for a discussion of this point, as well as pointing out some typos.
} 
allows for a more general solution,

$$
\Delta\left(\psi_{n}\right)=q n(k-n) / k+M_{n}
$$

(for $S U(2)$; for other algebras it is similar), where $\psi_{n}$ is the $n$th $Z_{k}$ parafermion, and $M_{n}$ are some integers. The multi-parafermions are for the large part, yet to be explored. However, we believe that the central charge is

$$
c(q)=q c(1) \bmod 4
$$

and thus by gluing them with free bosons it is possible to get the central charge of the pseudo affine systems. We hope to report on this in the future.

\section{ACKNOWLEDGEMENTS}

It is a pleasure to thank E. Baver for his participation in the early stages of this work.

\section{REFERENCES}

1. A.M. Polyakov, A.A. Belavin and A.B. Zamolodchikov, Nucl. Phys. B 241 (1984) 333.

2. E. Verlinde, Nucl. Phys. B 300 (1988) 360

3. D. Gepner, Caltech preprint CALT-68-1825, hepth-9211100.

4. E. Baver, D. Gepner and U. Gursoy, preprint hepth-9811100

5. D. Gepner and E. Witten, Nucl. Phys. B 278 (1986) 493.

6. V.A. Fateev and A.B. Zamolodchikov, Zh.Eksp.Theor.Fiz. 89 (1985) 215; D. Gepner and Z. Qiu, Nucl. Phys. B 285 (1987) 423; D. Gepner, Nucl. Phys. B 290 (1987) 10.

7. V.G. Kac, Infinite dimensional Lie algebras, Cambridge Univ. press, 1990. 